\title{
Noninferiority trials: What's clinically (ir)relevant?
}

Volkmar Falk, MD, a,b,c,d and Tim Friede, $\mathrm{PhD}^{\mathrm{e}, \mathrm{f}}$

The debates in clinical cardiovascular science are increasingly dominated by the results of noninferiority trials. Although there is no doubt that these trials are fundamental to generate the evidence on which we make our clinical decisions, it is equally important that clinicians clearly understand the principles, benefits, and limitations of the underlying trial design and statistics.

\section{WHY NONINFERIORITY TRIALS ARE USED}

When comparing 2 treatments in a randomized trial, typically the question is whether one treatment is superior to the other in terms of the primary efficacy end point. An example is the PARTNER 1B trial of transcatheter aortic valve replacement versus continued medical management for inoperable patients that showed transcatheter aortic valve replacement to be superior in terms of mortality. ${ }^{1}$ However, in the cardiovascular field, particularly when a new device is being compared with an already approved device of the same type, the trial's aim is to demonstrate similar effects on clinically relevant outcomes (efficacy or safety). For example, this may apply for a new transcatheter aortic valve that needs to be compared for efficacy with an established competitor. Because it is technically not possible to demonstrate that the 2 treatment effects are exactly the same (equivalent), we resort to the metric of similar effect size that only differs by a clinically irrelevant amount. Such trials are called "noninferiority trials." Usually the trial is designed to show that one treatment, such as a device, is no worse than another, apart from a clinically irrelevant amount, which is referred to as the "noninferiority margin."

The choice of the noninferiority margin is crucial in any noninferiority trial, and an inadequate margin will draw the design and conclusions from the study into question. If a

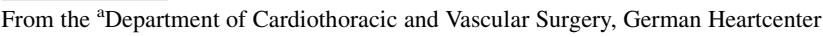
Berlin, Berlin, Germany; ${ }^{b}$ Department of Cardiovascular Surgery, Charite Universitätsmedizin Berlin, Berlin, Germany; ${ }^{\mathrm{c}}$ German Center for Cardiovascular Research (DZHK), partner site Berlin, Berlin, Germany; ${ }^{\mathrm{d}}$ Department of Health Sciences and Technology, Translational Cardiovascular Technologies, Swiss Federal Institute of Technology (ETH), Zurich, Switzerland; ${ }^{\mathrm{e} D e p a r t m e n t}$ of Medical Statistics, University Medical Center Göttingen, Göttingen, Germany; and ${ }^{\mathrm{f}}$ German Center for Cardiovascular Research DZHK, partner site Göttingen, Göttingen, Germany.

Received for publication Aug 5, 2019; revisions received March 20, 2020; accepted for publication March 24, 2020; available ahead of print May 31, 2020.

Address for reprints: Volkmar Falk, MD, Klinik für Herz-Thorax-Gefässchirurgie, Deutsches Herzzentrum Berlin, Augustenburger Platz 1, 13353 Berlin, Germany (E-mail: falk@dhzb.de,web: www.dhzb.de).

J Thorac Cardiovasc Surg 2021;161:2119-23

$0022-5223 / \$ 36.00$

Copyright (C) 2020 by The American Association for Thoracic Surgery

https://doi.org/10.1016/j.jtcvs.2020.03.168
}

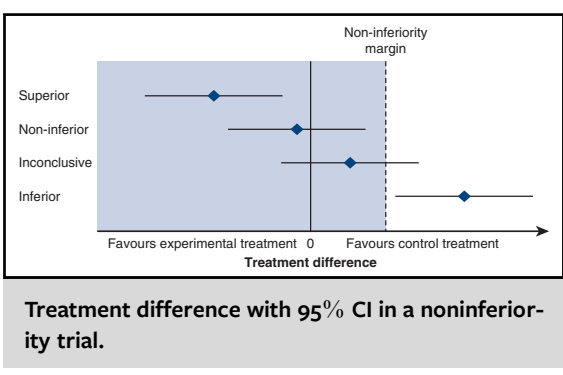

$$
\begin{aligned}
& \text { CENTRAL MESSAGE } \\
& \text { Noninferiority trials need to be } \\
& \text { interpreted with a good under- } \\
& \text { standing of the underlying } \\
& \text { applied statistics and good judg- } \\
& \text { ment with regard to the trial } \\
& \text { design. }
\end{aligned}
$$$$
\text { See Commentaries on pages } 2124 \text { and } 2125 .
$$

margin was chosen that is too large, usually the trial is too small to be meaningful, a problem that cannot be rectified in the analysis stage. The noninferiority margin must be prospectively defined in the study protocol. Setting an inappropriately wide margin can cause a noninferiority test to misleadingly conclude an inferior treatment to be effective. When trials are carried out in a regulatory context, the determination of the noninferiority margin needs to be set in the context of regulations issued by health authorities and the respective guidelines. ${ }^{2,3}$ Choosing a noninferiority margin is never simply a statistical or clinical matter, but always requires expertise in both. Before the margin can be set, the treatment effect scale needs careful consideration in terms of the clinical implications. For instance, interpretation might be easier on one scale than on another. With survival or more generally time-to-event data, often the hazard ratio is used as the effect scale. This might require some translation to survival or event probabilities at certain time points to be directly interpretable. For example, the currently ongoing randomized controlled DEDICATE trial comparing transcatheter aortic valve implantation and surgical aortic valve replacement uses a hazard ratio of 1.1 as a noninferiority margin for overall survival as its end point. ${ }^{4}$

If the objective of the noninferiority trial is to demonstrate clinical equivalence of 2 alternative therapies in terms 
of clinical efficacy and safety end points in the presence of an advantage of one of the therapies over the other with regard to another end point, then the question is which effects could be considered clinically irrelevant. If there is a good understanding of relevant effect sizes, then it is not uncommon to consider effects that are only half as large as clinically irrelevant. ${ }^{3}$ When dealing with end points such as mortality or major clinical events such as disabling strokes, the definition of irrelevant effect sizes can be challenging.

For the indirect comparison of an experimental therapy to placebo or sham therapy by comparison with a reference treatment, the effect of the reference therapy versus placebo or sham control is key. It is not uncommon to use a proportion (eg, 50\%) of the effect of the reference therapy as established in one or several randomized controlled trials versus placebo or sham control. ${ }^{3}$ If several trials exist, these are typically summarized by a meta-analysis yielding an effect estimate with confidence interval (CI). These trials have been typically conducted a number of years earlier; therefore, the assumption that the treatment effect observed in those historic trial populations would also apply to modern-day trial populations is crucial. The assumption is known as the constancy assumption, ${ }^{3}$ which is critical for determining the noninferiority margin.

\section{HOW TO TEST FOR NONINFERIORITY?}

In a trial intending to show that there is a difference less than a specific amount between control and experimental treatments, a noninferiority design statistically tests the null hypothesis that the experimental treatment is inferior by the noninferiority margin (Figure 1). Inference is commonly based on CIs; Figure 1 illustrates the process of drawing conclusions about noninferiority based on CIs for the treatment effect.

If noninferiority has been demonstrated by a certain margin, noninferiority tests with smaller margins or even superiority tests can be carried out at the same significance level $\alpha$ (usually $2.5 \% 1$-sided that corresponds to the standard 5\% 2-sided) while maintaining control of the overall type I error rate (sometimes referred to as "family-wise or experiment-wise type I error rate") at level $\alpha .^{5}$ The argument is that an a priori ordered hypotheses is tested (also referred to as "hierarchical testing procedure"). As soon as any of the null hypotheses in the hierarchy are true, the probability to reject this or any null hypothesis further down the hierarchy is at most $\alpha$.

In contrast to superiority trials, there is no clear preference for the intention-to-treat (ITT) principle versus per protocol (PP) or as-treated analyses in defining the primary analysis population. Whereas ITT analyses tend to be conservative in superiority trials biasing the treatment effect toward the null, this is not the case in noninferiority trials as no difference between the treatments is now the alternative and not the null hypothesis. The problem with PP or astreated analyses generally is that the analysis population is defined by postrandomization events, and therefore PP analyses, unlike ITT analyses, cannot rely on the randomization. Therefore, ITT and PP analyses both suffer from certain problems, but are typically considered to be on equal footing in noninferiority trials. This means that noninferiority needs to be demonstrated in both the PP and ITT analyses.

\section{PLANNING A NONINFERIORITY TRIAL}

By standard practice, patients are randomized in a ratio of $1: 1$ to the treatment groups. In superiority trials, this is optimal from a statistical point of view in the sense that the sample size is minimized to achieve a certain power. In noninferiority trials, however, this can be different, for instance when using relative rather than absolute noninferiority margins. For practical reasons, investigators might choose to deviate from the optimal allocation. This is the

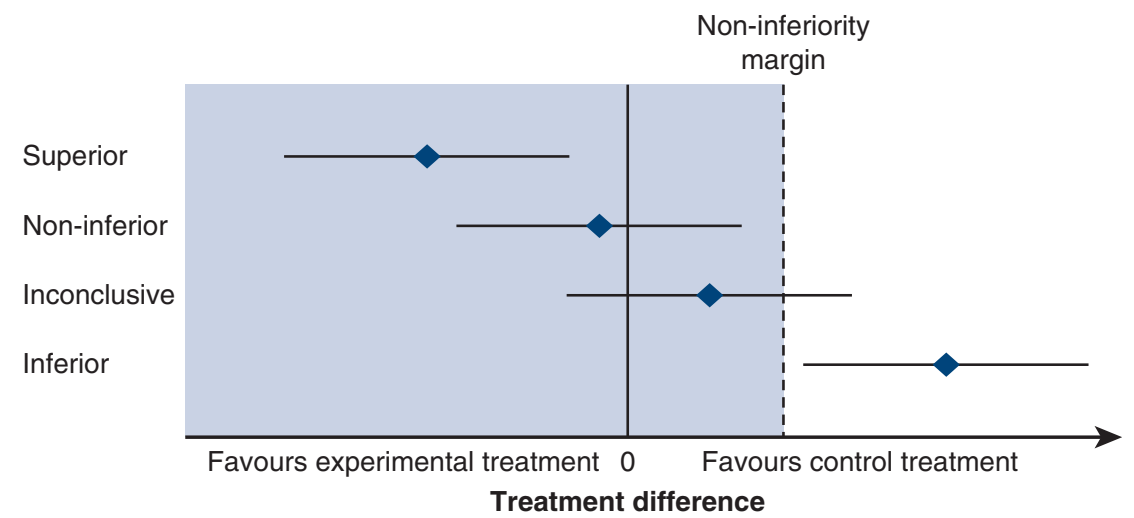

FIGURE 1. Hypothetical results of a noninferiority study illustrating the importance of the noninferiority margin in the interpretation. The schematic shows the treatment difference with $95 \%$ CI for 4 potential outcomes for the experimental treatment in comparison with the control treatment including superiority, noninferiority, inconclusive results, and inferiority. The hypothesis is the area to the right of the noninferiority margin. The alternative hypothesis that the experimental treatment is inferior by at most the noninferiority margin is the area to the left of the noninferiority margin marked in blue. 
case sometimes in superiority trials when the decision is taken to randomize not $1: 1$ but to allocate more patients to the experimental treatment. For instance, patients are randomized 2:1 to exercise intervention or standard of care in the in the ongoing Ex-VAD trial. ${ }^{6}$ This makes the trial more attractive to patients and treating physicians as more patients benefit from the additional, potentially efficacious intervention. Similar arguments would also apply in the context of noninferiority trials of course.

The choice of the primary end point is a crucial step in any trial. In cardiac surgery, a number of clinical events are often relevant; therefore, it is not uncommon to combine these in a composite end point. It is good practice to consider the individual components of the composite as secondary end points because they are usually of varying clinical importance. This is of particular importance in noninferiority trials, because apparent noninferiority in terms of the primary composite end point actually might have been achieved at the cost of a redistribution of the events toward more severe event types.

An overview of sample size calculations for cardiovascular trials has been published. ${ }^{7}$ The main difference between sample size calculations for noninferiority trials and superiority trials is that the assumed treatment effect in the calculations is replaced by the difference between the assumed treatment effect and the noninferiority margin. Commonly, the assumption is made that the experimental treatment and the control have similar effects. Therefore, the treatment effect in the sample size calculation for the superiority trial is effectively replaced by the noninferiority margin. As explained earlier, the noninferiority margin, a clinically irrelevant effect, is often half the size of clinically relevant effects. Therefore, as a rule of thumb, noninferiority trials tend to be 4 times larger than superiority trials. ${ }^{7}$

The planning of any trial relies on a number of assumptions. With continuous end points, for example, the variability of the measured end point needs to be specified to calculate the required sample size. Adaptive designs with sample size re-estimation have been proposed to mitigate the risks of an inadequate sample size. If only a nuisance parameter such as the variance is estimated during the course of the study, it is not necessary to unblind the study. Such reassessments of the sample size can be carried out on the basis of noncomparative data, that is, without knowledge of the patients' treatment group allocation..$^{8-10}$ In blinded trials, these procedures are referred to as "blinded sample size re-estimation"; sometimes the term is also used in the context of open trials. ${ }^{11}$ Although adaptations based on noncomparative data generally do not inflate the probability of a type I error (falsely significant result), the error probability is inflated in noninferiority trials although by only a small, often negligible amount. $^{8}$

\section{WHAT ARE THE RISKS}

As discussed earlier, choosing a noninferiority margin that is unacceptably large poses one of the biggest threats. In this section, we discuss briefly other issues that require careful consideration.

Assay sensitivity is the "ability to distinguish an effective treatment from a less effective or ineffective treatment." 12 In 2-arm noninferiority trials, "assay sensitivity" has to be assumed and cannot be checked. Therefore, sometimes so-called gold standard designs are used. ${ }^{13}$ These are 3 -arm designs including the experimental treatment and active as well as placebo or sham control. The optimal treatment allocation usually favors the active arms, that is, the experimental therapy and the active control. ${ }^{13}$

The purpose of blinding in clinical trials is the minimization of biases in the treatment of patients and the assessment of the outcomes. Whereas double-blinding is the gold standard in drug trials, blinding is often not feasible in surgery trials. In such circumstances, the blinded assessment of the outcomes is of particular importance. ${ }^{14}$ This can, for example, be achieved by a clinical events committee or core laboratory. In general, blinding is an effective measure to minimize bias in superiority trials. Noninferiority trials are less robust for risk of bias despite blinding. This is illustrated by a hypothetical and admittedly extreme example: In a noninferiority trial with an investigator-assessed outcome, an investigator tends to rate the outcomes for all patients similarly. This will bias the treatment effect toward no difference and at the same time reduce the variability of the outcomes. This combination will make a false proof of noninferiority more likely. This type of bias is more likely with soft end points and cannot occur with hard end points such as death.

\section{HOW TO REPORT A NONINFERIORITY TRIAL}

Because the noninferiority margin plays such an important role, it should be reported up-front and not be hidden away in the statistics section of any report or publication. Also, a rationale for the choice of the margin should be provided. An extension of the CONSORT Statement provides specific guidance on how to report noninferiority trials. ${ }^{15}$

\section{EXAMPLE}

The Synergy between PCI with Taxus and Cardiac Surgery (SYNTAX) trial was a noninferiority randomized controlled trial to compare the outcomes of percutaneous coronary intervention (PCI) with coronary artery bypass grafting $(\mathrm{CABG})$ in patients with multivessel coronary artery disease and left main (LM) coronary artery disease. The primary combined clinical end point was defined as the major cardiovascular or cerebrovascular event rate at 12 months. The major cardiovascular or cerebrovascular event probabilities at 12 months were $12.4 \%$ and $17.8 \%$ 
in the CABG and PCI groups, respectively, resulting in a treatment group difference of 5.5 percentage points with an upper $95 \%$ confidence limit of 8.3 percentage points. ${ }^{16}$ The prespecified noninferiority margin was set at 6.6 percentage points, with a 1 -sided $95 \%$ upper confidence limit for the difference. Because the upper confidence limit of the difference for the primary end point was with 8.3 percentage points larger than the margin of 6.6 percentage points, PCI with drug-eluting stents failed to show noninferiority. ${ }^{16}$ Note that in the SYNTAX trial, a 1-sided 95\% confidence limit was used, which is narrower than the usual 2-sided $95 \%$ confidence limit, which corresponds to 1-sided 97.5\% limits.

Prespecified margins were used to test the 3-vessel disease and LM subgroups, but because noninferiority was not proven in the overall cohort, specific information for each of these outcomes could only be considered observational in nature and hypothesis generating, which the authors rightfully stated in the original publication. ${ }^{16}$ If the primary end point is not met, subgroup analyses of components of the primary end point or analyses of subcohorts of the trial population are scientifically often not sound. ${ }^{17}$

Despite the hierarchical nature of the primary end point analysis in the SYNTAX trial, and the fact that noninferiority was not met for the primary end point, further analyses were subsequently performed. In addition, the prespecified margin for noninferiority for the LM subgroup in the SYNTAX trial was set unusually high at $9 \%$ with a 1 -sided $5 \%$ significance level (Supplemental Appendix in Serruys and colleagues ${ }^{16}$ ). The LM cohort of SYNTAX was then even further stratified into subgroups according to a SYNTAX score claiming equipoise for both treatments for patients with a SYNTAX score of less than or equal to 32 up to 5 years with an odds ratio of $0.94(0.67-1.33 ; P=.74) .^{18}$ In the "Limitations" section, the authors highlight that hierarchical primary end point testing of the SYNTAX study did allow testing of the LM subgroup only if the overall comparison had reached statistical significance, which it did not. Thus, the authors stated that the results "should be interpreted with care." The analysis of clearly underpowered LM subgroups from a negative noninferiority trial nevertheless led to a class 2A level B recommendation for PCI in patients with LM disease and a SYNTAX score of 23 to 32 in the 2014 European Society of Cardiology/European Association for Cardio-Thoracic Surgery Myocardial Revascularization Guidelines. ${ }^{19}$ This, despite the fact that not a single adequately powered randomized controlled trial justifying a Level A or B recommendation had ever been performed at this point in time.

It was pointed out earlier that the general enthusiasm for trial evidence may be harmful when subgroup analyses are carried out without a proper appreciation of the statistical pitfalls and that undue emphasis on a particular subgroup may result in inappropriate treatment. ${ }^{20,21}$ The now available results of 2 randomized controlled trials in patients with LM disease (EXCEL and NOBLE) have consistently demonstrated the benefits of CABG versus PCI with longer follow-up. ${ }^{22,23}$

\section{CONCLUSIONS}

Noninferiority trials have their firm place in clinical research and impact clinical decision making. However, they are not without limitations, and the interpretation of these trials must be done with good judgment on the trial design.

\section{Conflict of Interest Statement}

Dr Friede has a financial relationship with AstraZeneca, Bayer, Biosense Webster, Boehringer Ingelheim, CSL Behring, Daiichi-Sankyo, Enanta, Fresenius Kabi, Galapagos, Janssen, Novartis, Penumbra, Roche, and Vifor. Dr Falk reports institutional financial activities outside the submitted work with following commercial entities: Medtronic $\mathrm{GmbH}$ (Meerbusch, Germany), Biotronik SE \& Co. (Berlin, Germany), Abbott GmbH \& Co. KG (Wiesbaden, Germany), Boston Scientific (Ratingen, Germany), Edwards Lifesciences (Unterschleissheim, Germany), Berlin Heart (Berlin, Germany), Novartis Pharma GmbH (Nuremburg, Germany), JOTEC GmbH (Hechingen, Germany), Zurich Heart (Zurich, Switzerland) in relation to educational grants (including travel support), fees for lectures and speeches, fees for professional consultation, and research and study funds.

The Journal policy requires editors and reviewers to disclose conflicts of interest and to decline handling or reviewing manuscripts for which they may have a conflict of interest. The editors and reviewers of this article have no conflicts of interest.

\section{References}

1. Leon MB, Smith CR, Mack M, Miller DC, Moses JW, Svensson LG, et al; PARTNER Trial Investigators. Transcatheter aortic-valve implantation for aortic stenosis in patients who cannot undergo surgery. N Engl J Med. 2010;363:1597-607.

2. Committee for Medicinal Products (CHMP) Guideline on the choice of the noninferiority margin. Stat Med. 2006;25:1628-38.

3. Food and Drug Administration (FDA) Guidance Document Non-Inferiority Clinical Trials 2016. Available at: https://www.fda.gov/regulatory-information/ search-fda-guidance-documents/non-inferiority-clinical-trials. Accessed June $12,2019$.

4. Seiffert M, Walther T, Hamm C, Falk V, Frey N, Thiele H, et al. The DEDICATE Trial: An independent all-comers trial of transcatheter aortic valve implantation vs. surgical aortic valve replacement in patients at low to intermediate operative risk is recruiting patients. Eur Heart J. 2019;40:331-3.

5. The European Agency for the Evaluation of Medicinal Products Evaluation of Medicines for Human Use. Points to consider on switching between superiority and non-inferiority 2000. Available at: https://www.ema.europa.eu/en/ documents/scientific-guideline/points-consider-switching-between-superioritynon-inferiority_en.pdf. Accessed June 12, 2019.

6. Friede T. Clinical trial design: statistical issues. In: Camm AJ, Lüscher TF, Maurer G, Serruys PW, eds. ESC CardioMed. 3rd ed. Oxford, UK: Oxford University Press; 2018.

7. Friede T, Kieser M. Blinded sample size reassessment in non-inferiority and equivalence trials. Stat Med. 2003;22:995-1007. 
8. Friede T, Kieser M. Blinded sample size re-estimation in superiority and noninferiority trials: bias versus variance in variance estimation. Pharm Stat. 2013;12:141-6.

9. Friede T, Mitchell C, Müller-Velten G. Blinded sample size reestimation in noninferiority studies with binary endpoints. Biom J. 2007;49:903-16.

10. Food and Drug Administration (FDA). Guidance Document Adaptive Designs for Clinical Trials of Drugs and Biologics Guidance for Industry 2019. Available at: https://www.fda.gov/regulatory-information/search-fda-guidance-documents/adaptivedesign-clinical-trials-drugs-and-biologics-guidance-industry. Accessed June 12, 2019.

11. Bobenko A, Schoenrath F, Knierim JH, Friede T, Verheyen N, Mehra MR, et al. Exercise training in patients with a left ventricular assist device (Ex-VAD): rationale and design of a multicentre, prospective, assessor-blinded, randomized, controlled trial. Eur J Heart Fail. 2019;9:1152-9.

12. ICH Topic E 10 Choice of Control Group in Clinical Trials (CPMP/ICH/364/96) 2001. Available at: https://www.ema.europa.eu/en/ich-e10-choice-controlgroup-clinical-trials. Accessed June 12, 2019.

13. Mütze T, Konietschke F, Munk A, Friede T. A studentized permutation test for three-arm trials in the 'gold standard' design. Stat Med. 2017;36:883-98.

14. Day SJ, Altman DG. Blinding in clinical trials and other studies. BMJ. 2000;321: 504.

15. Piaggio G, Elbourne DR, Altman DG, Pocock SJ, Evans SJ. Reporting of noninferiority and equivalence randomized trials: an extension of the CONSORT statement. JAMA. 2006;295:1152-60.

16. Serruys PW, Morice MC, Kappetein AP, Colombo A, Holmes DR, Mack MJ, et al; SYNTAX Investigators. Percutaneous coronary intervention versus coronary-artery bypass grafting for severe coronary artery disease. $N$ Engl $J$ Med. 2009;360:961-72.

17. Tanniou J, van der Tweel I, Teerenstra S, Roes KCB. Estimates of subgroup treatment effects in overall nonsignificant trials: to what extent should we believe in them? Pharm Stat. 2017;16:280-95.

18. Morice MC, Serruys PW, Kappetein AP, Feldman TE, Ståhle E, Colombo A, et al. Five-year outcomes in patients with left main disease treated with either percutaneous coronary intervention or coronary artery bypass grafting in the synergy between percutaneous coronary intervention with taxus and cardiac surgery trial. Circulation. 2014;129:2388-94.

19. Kolh P, Windecker S, Alfonso F, Collet JP, Cremer J, Falk V, et al. 2014 ESC/ EACTS Guidelines on myocardial revascularization. Eur J Cardiothorac Surg. 2014;46:517-92.

20. Sleight P. Debate: subgroup analyses in clinical trials: fun to look at - but don't believe them! Curr Control Trials Cardiovasc Med. 2000;1:25-7.

21. Ruel M, Falk V, Farkouh ME, Freemantle N, Gaudino MF, Glineur D, et al Myocardial Revascularization Trials. Circulation. 2018;138:2943-51.

22. Holm NR, Mäkikallio T, Lindsay MM, Spence MS, Erglis A, Menown IBA, et al NOBLE investigators. Percutaneous coronary angioplasty versus coronary artery bypass grafting in the treatment of unprotected left main stenosis: updated 5-year outcomes from the randomised, non-inferiority NOBLE trial. Lancet. 2019;395:191-9.

23. Stone GW, Kappetein AP, Sabik JF, Pocock SJ, Morice MC, Puskas J, et al EXCEL Trial Investigators. Five-year outcomes after PCI or CABG for left main coronary disease. N Engl J Med. 2019;38:1820-30. 